\title{
Splenic Abscess: A Rare Complication of Sleeve Gastrectomy: A Case Report and literature Review.
}

\author{
Abbas AR Mohamed ${ }^{1 *}$, Turki Atia Al Quarshi², Sarah Abbas Mohamed ${ }^{3}$ \\ ${ }^{1}$ Consultant General and Laparoscopic Surgeon, Department of Surgical Specialties,NGH -Madinah -KSA. \\ ${ }^{2}$ Consultant Laparoscopic, obesity and metabolic Surgery, Department of Surgical Specialties, NGH -Madinah -KSA.
}

${ }^{3}$ Specialty training registrar, general surgery, Wrexham Maelor Hospital-Wrexham -UK.

*Corresponding author: Abbas AR Mohamed, Consultant General and Laparoscopic Surgeon, Department of Surgical Specialties, NGH Madinah -KSA.

Received date: January 22, 2020; Accepted date: January 27, 2020; published date: January 31 , 2020

Citation: Abbas ARM, Turki Atia AQ, Sarah AM (2020) Splenic Abscess: A Rare Complication of Sleeve Gastrectomy: A Case Report and literature Review. J Clinical Research and Reports, 2(3); DOI:10.31579/2690-1919/019

Copyright: (C) 2020 Abbas AR Mohamed. This is an open access article distributed under the Creative Commons Attribution License, which permits unrestricted use, distribution, and reproduction in any medium, provided the original work is properly cited.

\section{Abstract}

Splenic abscess is a rare complication of laparoscopic sleeve gastrectomy (LSG) with only a few cases reported in the literature. Here we report a 41-year-old healthy female with a BMI of 42 who had laparoscopic sleeve gastrectomy complicated with splenic vein thrombosis and splenic infarctions in the early postoperative period and progressed to a splenic abscess with a latediagnosed leakage from the gastric remnant. She was treated with laparoscopic splenectomy and a Roux-en-Y esophagojejunostomy.

Keywords: Sleeve gastrectomy; splenic infarction; gastric leak; splenic abscess

\section{Introduction}

Laparoscopic sleeve gastrectomy (LSG) is a well-established standard procedure to treat obesity. Although many complications were reported with LSG, the splenic abscess is a rare outcome and only a few cases have been reported. We report a case of a delayed post LSG splenic abscess due to a progression of an early postoperative splenic infarction. we further review the literature with the etiology, presentation, and management of this rare complication.

\section{Case presentation}

A 41 years old healthy obese lady with a BMI of 42 was admitted for sleeve gastrectomy. She had no comorbidities and all perioperative investigations were normal. She was started on the perioperative DVT prophylaxis the night before surgery and antibiotic prophylaxis just before surgery as per our protocol. She had straight forwards sleeve gastrectomy without excessive bleeding or apparent leakage.

The surgical technique involved the separation of the greater omentum from the stomach using Ligasure starting from the pylorus and proceeding upward to the gastroesophageal junction keeping as much as possible nearer to the stomach wall. The calibration was done with a 38-Fr calibration boogie and stapling was done with Endo GIA stapler (Covidien, USA) starting $6 \mathrm{~cm}$ from the pylorus and continued up to the gastroesophageal junction using black and purple staples without reinforcement sutures to the staple line. Methylene blue dye was used to check for Intraoperative leakage at the end of the procedure.

The patient developed extensive ecchymosis around the camera port and her hemoglobin was dropped significantly ( 3 gram \%) with two spikes of fever in the second post-operative day although she remained hemodynamically stable. The heparin was stopped and a gastrograffin study showed free passage of the contrast through the gastric sleeve without any obvious signs of leaking, stasis or narrowing. (Figure 1).

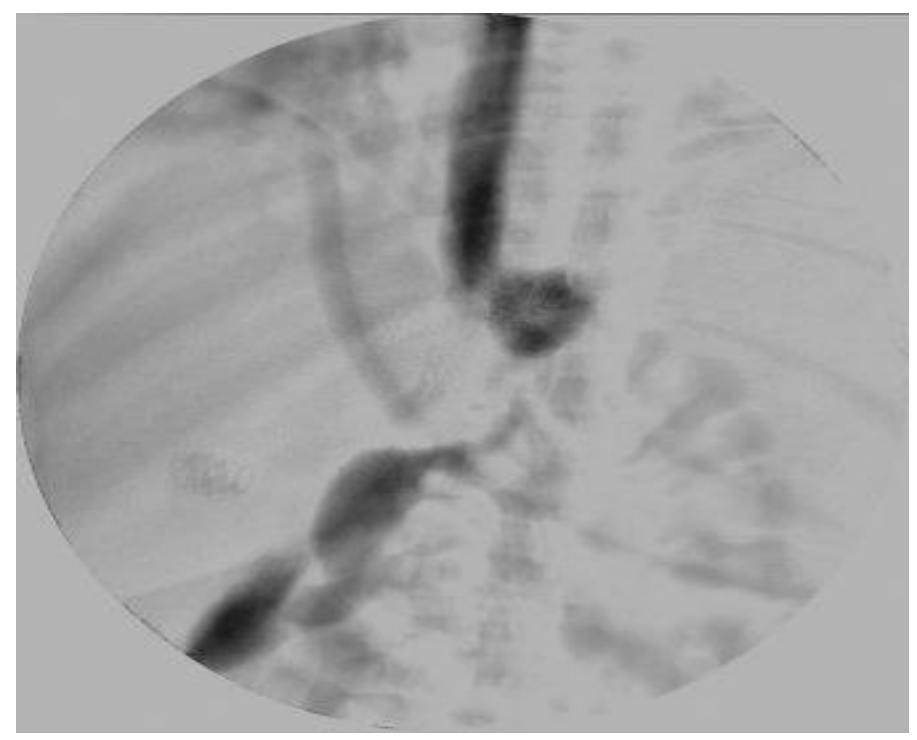

Figure 1: Gastrograffin study showing passage of the contrast through the gastric sleeve without any obvious signs of leaking, stasis or narrowing.

She also had a chest and abdominal CT scan which showed mild-tomoderate left pleural effusion with left lung base consolidation on chest images (figure 2A) and large splenic infarction involving more than 50\% of the spleen (Figures 2A-2B) with distal splenic vein thrombosis (Figures $3 \mathrm{~B}-3 \mathrm{C}$ ) together with a localized hematoma (about $15 \mathrm{ml}$ ) anterior to the 
pancreas at the inferior aspect of the lesser sac and mild free fluid in abdomen and pelvis on abdominal images. There was No obvious leakage of oral contrast was demonstrated in all images. The patient was started on antibiotics and therapeutic dose of enoxaparin.

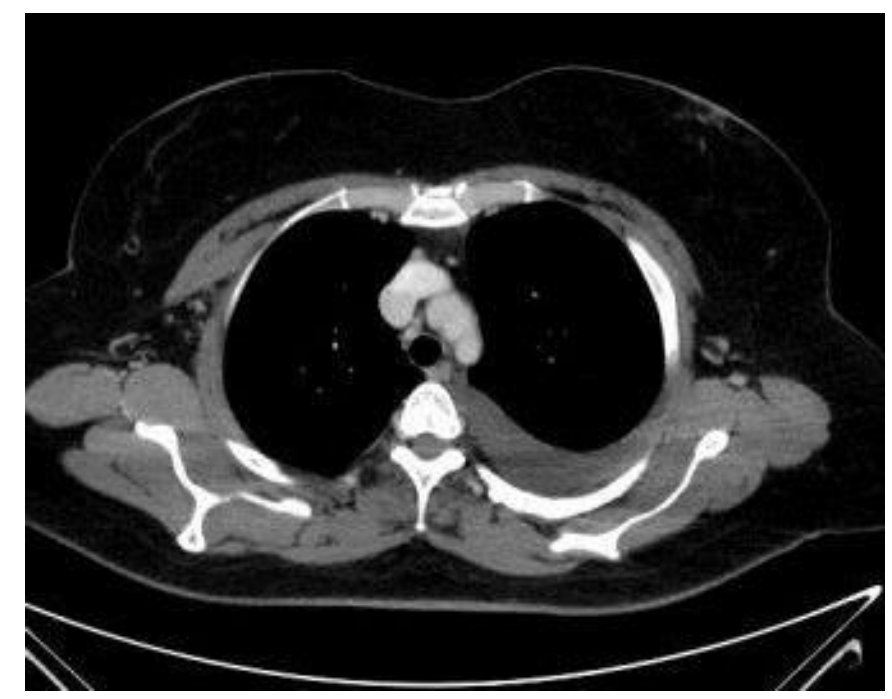

Figure 2A: CT image showing mild to moderate left pleural effusion.

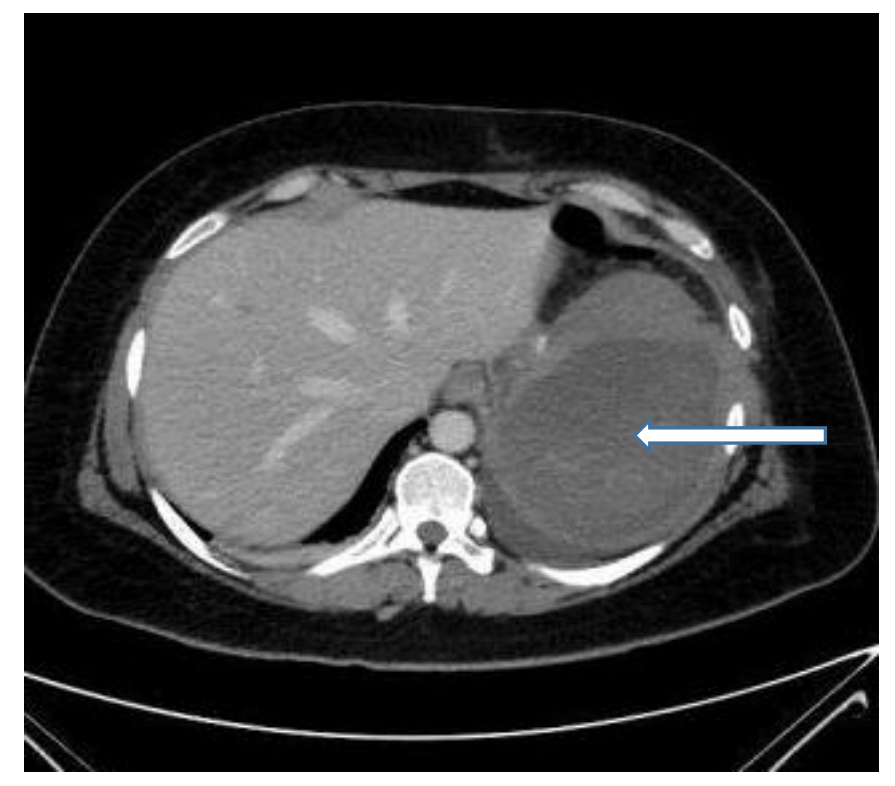

Figure 2B: CT image showing a large splenic infarction involving more than $50 \%$ of the spleen (the arrow).

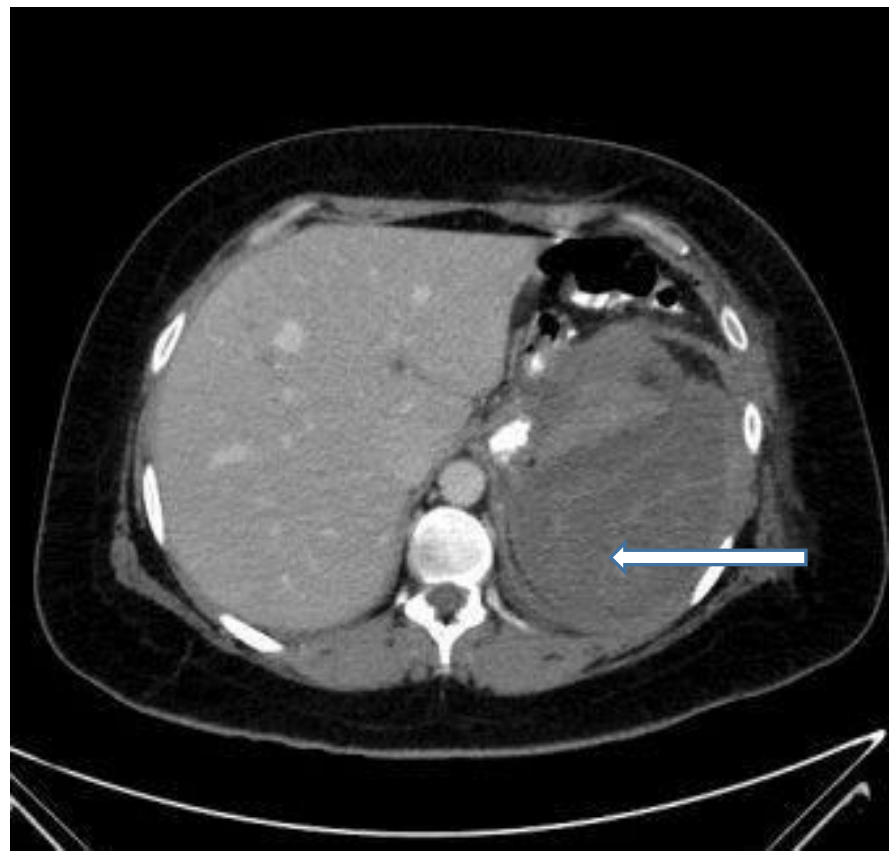

Figure 2C: CT image at a different level showing the same findings of figure $2 \mathrm{~B}$.

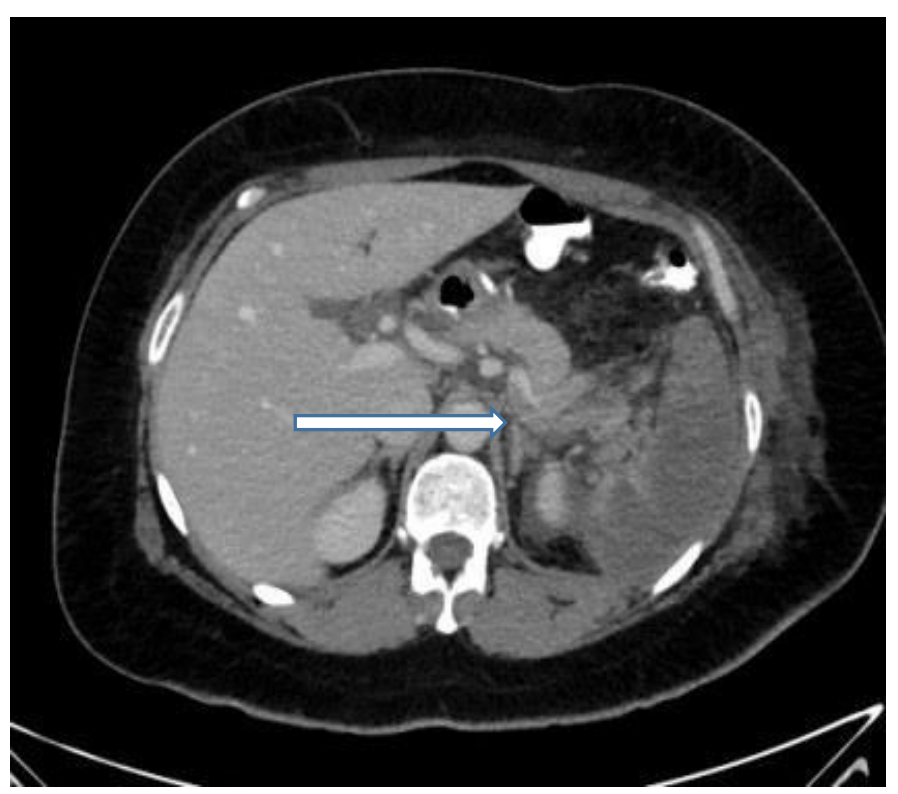

Figure 3A: CT image (axial view) showing a non-occlusive thrombus within the splenic vein (the arrow). 


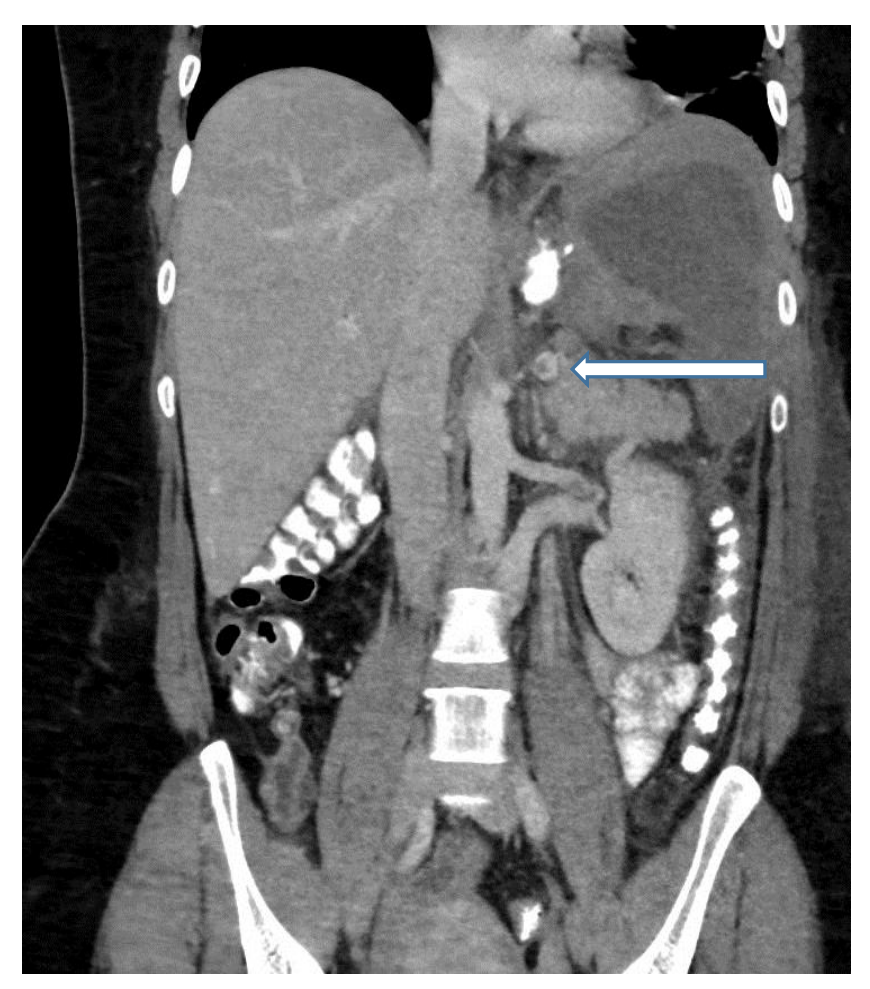

Figure 3B: CT image (coronary view) showing the same findings of figure $3 \mathrm{~A}$ (the arrow).

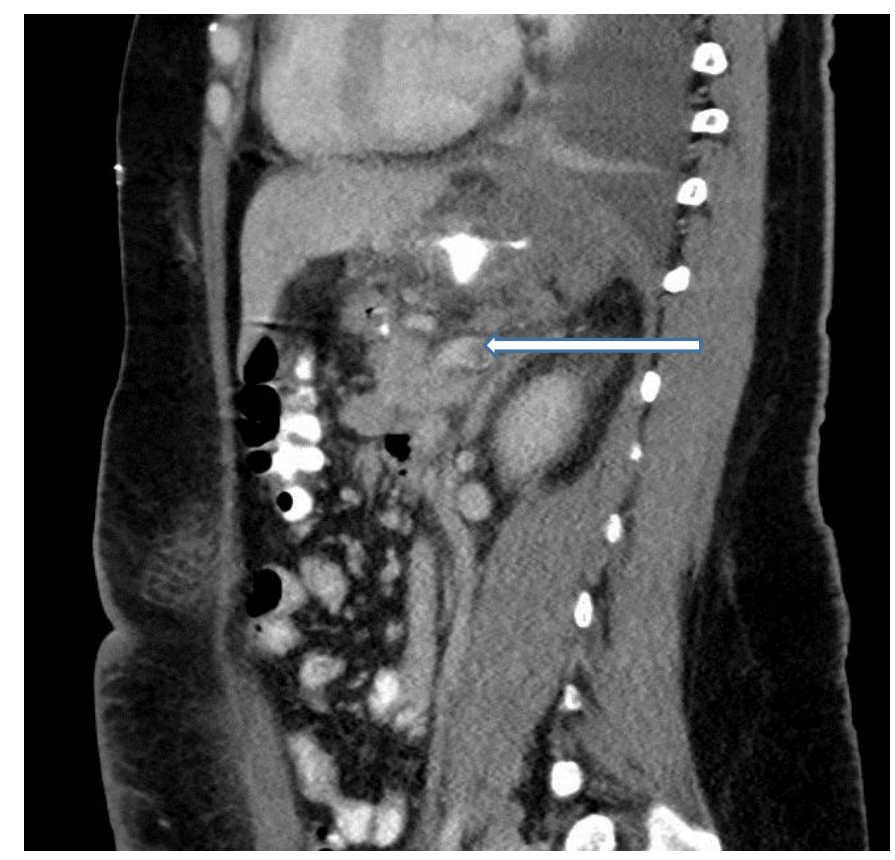

Figure 3C: CT image (sagittal view) showing the same findings of figure $3 \mathrm{~A}$ (the arrow).

In view of the persistent fever and increasing left-sided pleural effusion, she had ultrasound-guided drainage of the pleural effusion with a drainage of about $500 \mathrm{ml}$ of clear fluids, however, follow up chest x-rays didn't show significant improvement and a chest drainage tube was inserted under LA (figure 4A,4B).

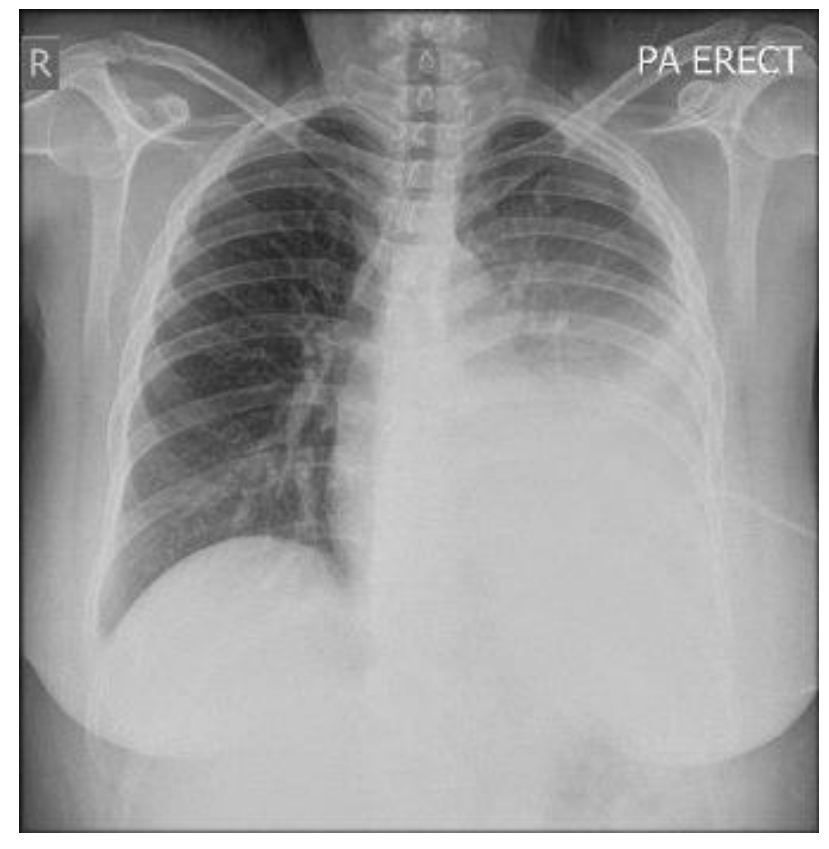

Figure 4A: Post ultrasound guided drainage of the left pleural effusion chest $\mathrm{x}$ ray showing the drainage tube in situ.

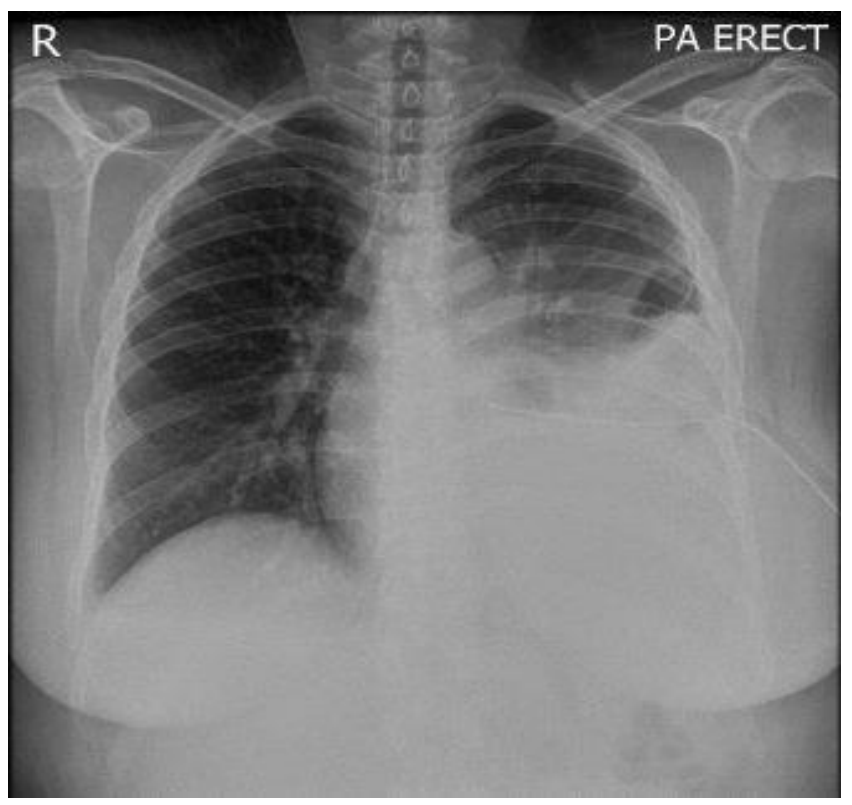

Figure 4B: Chest $x$ ray after chest drain insertion with mild reduction in the size of the left pleural effusion.

The patient was commenced on chest physiotherapy, intravenous antibiotic and shifted to oral anticoagulant. Although she remained well and tolerated oral fluids, she continued to have on and off fever.

A repeat CT scan 3 weeks after surgery showed moderate left-sided pleural effusion. The spleen is almost totally replaced by hypodensity (fluid density) with multiple air pockets and an air-fluid level anteriorly. There was also some peri-splenic fat stranding and minimal fluid noted on the lateral aspect of the upper pole of the left kidney. The CT features of the spleen are consistent with splenic abscess (figures $5 \mathrm{~A}, 5 \mathrm{~B}$ ). 


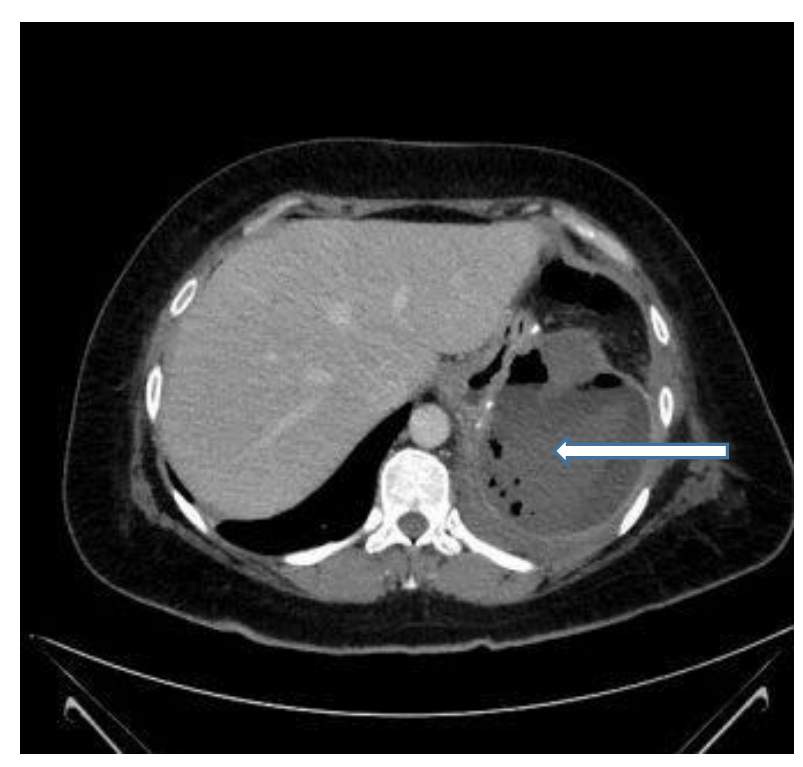

Figure 5A and 5B: CT scan images showing that the spleen is almost totally replaced by fluid hypodensity with multiple air pockets and an airfluid level, features were highly suggestive of splenic abscess.

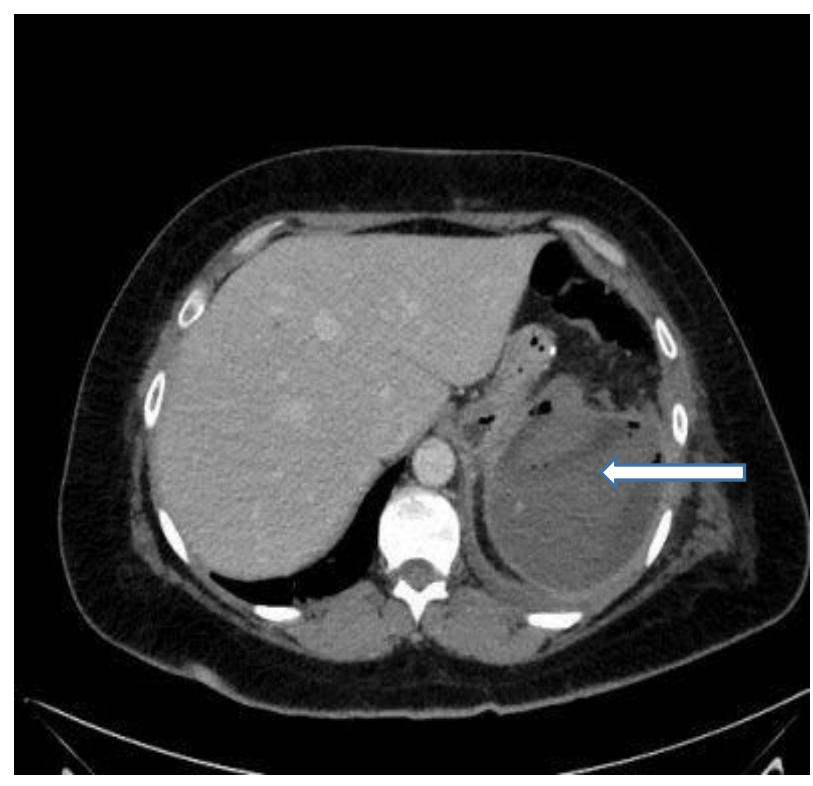

Figure 5B: CT scan image at a different level showing the same findings of figure $5 \mathrm{~A}$.

Because of the CT scan findings which were highly suggestive of splenic abscess, the decision was made for laparoscopic exploration, splenectomy, and intra-operative gastroscopy.

On laparoscopic exploration, there was huge splenic abscess extending to the left subdiaphragmatic area and the spleen was found to be converted into a bag of pus. (Figure 6,7)

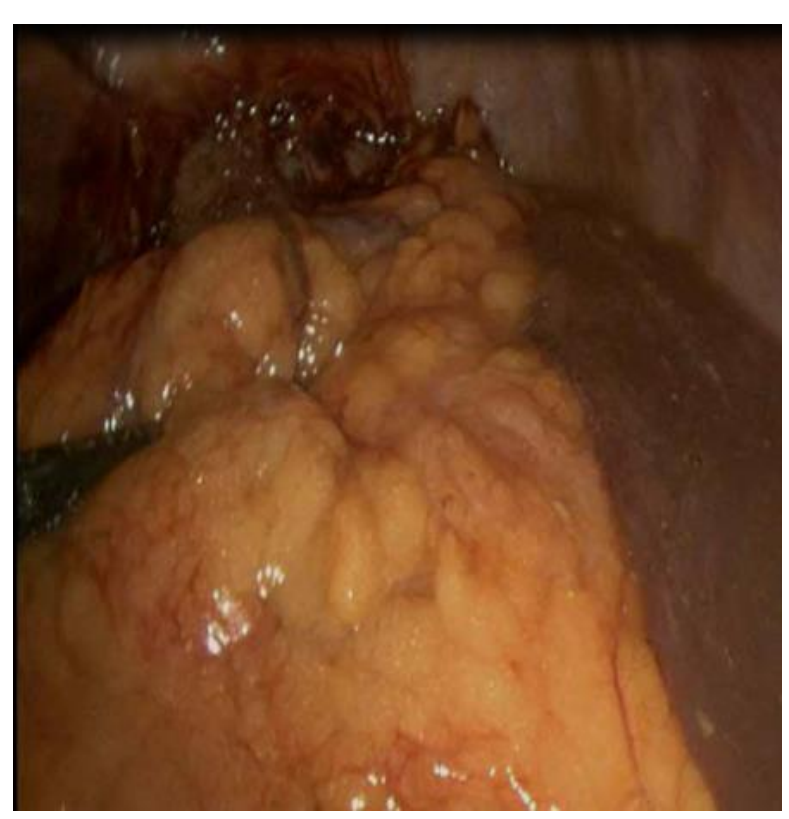

Figure 6: showing a huge splenic abscess extending to the left subdiaphragmatic area.

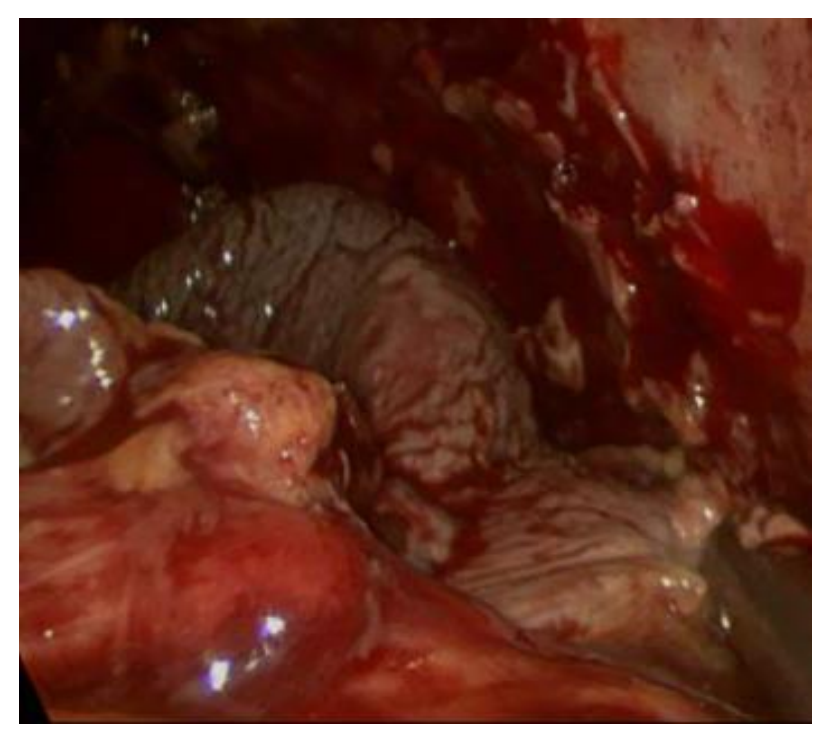

Figure 7: showing that the spleen was converted into a bag of pus.

The intraoperative gastroscopy and intraoperative testing for leakage demonstrated a leakage from small defect about half $\mathrm{cm}$ just below the gastro-oesophageal junction. splenectomy and a Roux-en-Y esophagojejunostomy (Figure 8 and 9) were performed together with washout and drainage of the left subdiaphragmatic space and the pelvis. The patient was kept for 48 hours in the intensive care unit and then transferred to the general wards. She made an uneventful recovery and discharged on the 7 th postoperative day. 


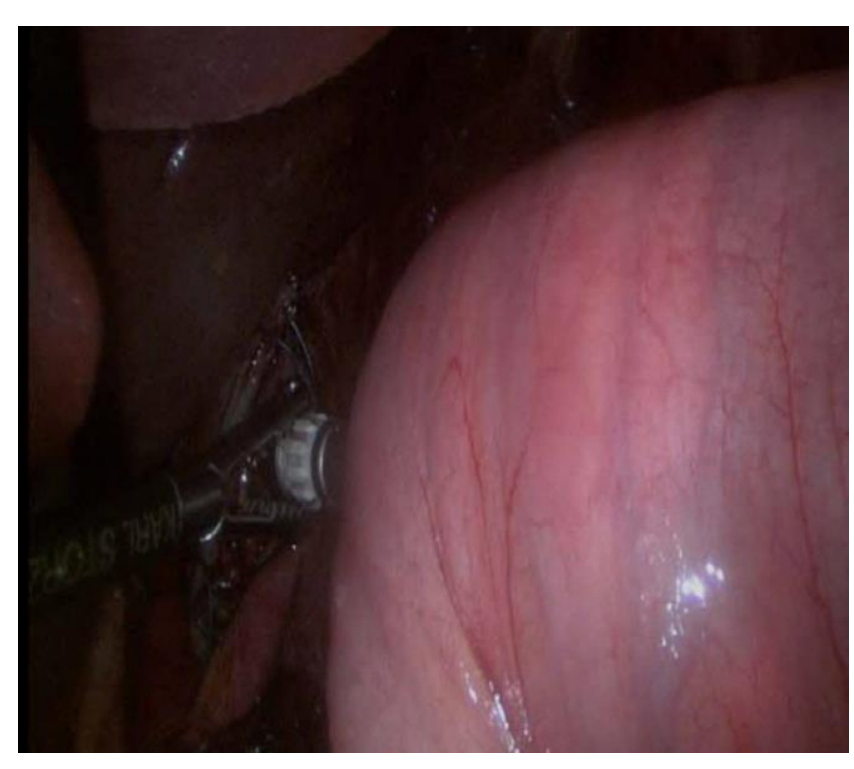

Figure 8: Showing step of the laparoscopic Roux-en-Y esophagojejunostomy with the anvil of the circular stapler in the esophagus.

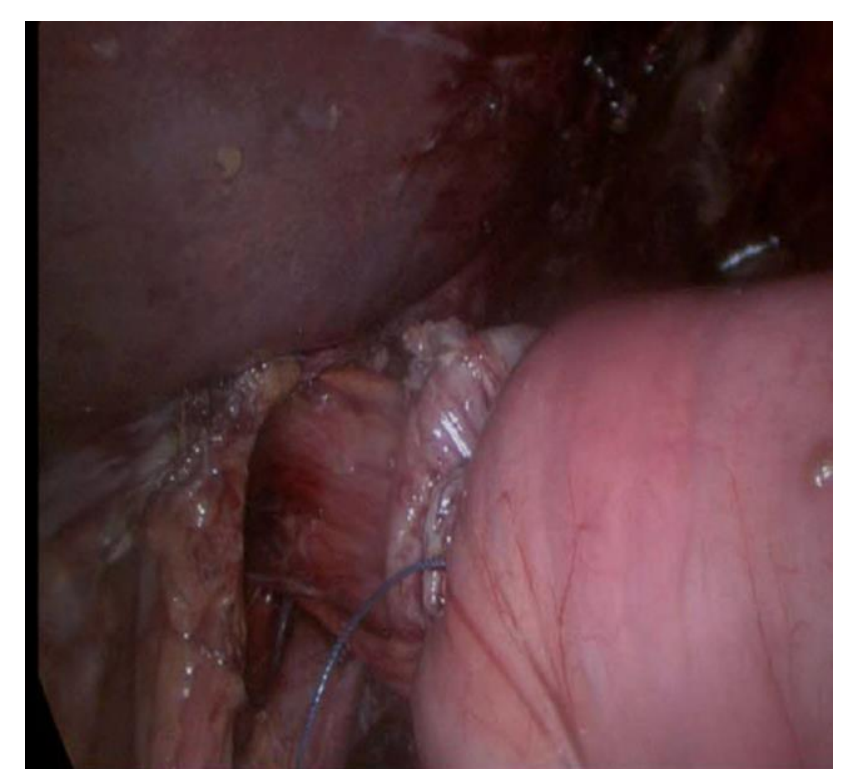

Figure 9: Showing the esophagojejunal anastomosis.

\section{Discussion:}

Laparoscopic sleeve gastrectomy (LSG), first described in 1988 as the initial restrictive component of the duodenal switch procedure [1], is now well established as one of the standard procedures for the surgical treatment of obesity. Its simplicity and low-risk profile make it more appealing [2] and the most commonly performed bariatric surgery procedure worldwide $[3,4]$. Although many complications were reported after LSG including staple line leakage, bleeding, sleeve stricture, delayed gastric emptying, gastric dilation, intra-abdominal abscess, trocar site hernia, trocar site infection, and splenic injury [5-8], the splenic abscess is extremely rare with only a few cases reported in the literature. Splenic demarcation and infarction of the upper pole is not uncommon and has been reported following LSG $[9,10]$. The reported incidences of splenic infarction after LSG is about $4.1 \%$ [11]. However, most of the reported cases were not symptomatic. Occlusion of the main splenic artery or vein alone seldom produces splenic infarction [12]. and the exact mechanism of post sleeve gastrectomy splenic infarction is not well known. It may be a result of a combination of factors including division of the short gastric vessels (SGVs) during LSG, splenic vein thrombosis, and upper terminal splenic artery branches division during the devascularization of the greater curvature of the stomach. Dhanasopon et al [13] suggest that the division of main branches of the splenic artery in close to the SGVs when the splenic artery divides before reaching the hilum of the spleen and when those branches constitute the main segmental arterial supply is the main contributory factor. We believe the combination of division of the SVG's and splenic vein thrombosis is the main cause of splenic infarction in our presented case. Splenic infarction may have no clinical relevance in the vast majority of patients [14-16] and it usually resolved spontaneously. When symptomatic, it often presents with vague or nonspecific signs and symptoms, including left upper quadrant pain, fever, nausea and vomiting, associated with leukocytosis and may be difficult to differentiate from the leakage [17]. More seriously it can rarely progress to a splenic abscess in the presence of a gastric leak or systemic infection as the case in our patient.

Splenic abscess is a rare clinical entity with an incidence of $0.2-0.7 \%$ in autopsy-based studies [18], with about 600 cases have been described so far in the international literature [19]. The vast majority of the available literature consisting of case reports and series from tertiary care centers [20]. Splenic abscess as a complication of laparoscopic sleeve gastrectomy (LSG) is even rarer with only 6 cases reported in the literature $(2,20-22)$.

Shanti, et al [2] reported a case similar to our case of post sleeve splenic infarction progressed to a splenic abscess in the presence of gastrectomy Leakage at the gastroesophageal junction in a 34 years old immunocompetent male presented four months after sleeve gastrectomy treated with a metallic stent and percutaneous drainage of the abscess.

Miguel E et al [20] reported another case of splenic abscess in a 45-yearold immunocompetent female developed 20 days after LSG without documented gastrostomy leaking. The patient was successfully treated with a combination of antibiotics and percutaneous drainage.

Avulov V et al [21] reported the first case of splenic abscess as a progression of splenic infarction after routine sleeve gastrectomy in a 19year-old healthy male two weeks after the surgery. The patient was treated with splenectomy.

Sakran N et al [2] reported two cases of post sleeve gastrectomy splenic abscess in a 36-year-old female two months after surgery treated with open splenectomy and another case in a 35-year-old female two and a half months after surgery treated initially with percutaneous drainage and antibiotic and subsequently with laparoscopic drainage.

Yardesh Singh et al [22] reported a case of delayed splenic abscess occurred 10 weeks after sleeve gastrectomy in a 44-year-old healthy man without an obvious leaking from the sleeve staple lines. The patient was treated with open splenectomy due to hemodynamic instability.

The exact cause of post sleeve splenic abscesses is not well known. However, many factors were suggested including a direct extension from a gastric staple-line leaking [21], and inadvertent splenic ischemia occurred after sleeve gastrectomy and iatrogenic splenic injury at the time of surgery resulting in devitalization and subsequent bacterial colonization [14]. 
Sakran et al [2] theorized that later infection of the splenic infarct, either via direct extension, from a nearby infection, or through hematological spread is the possible mechanism for its occurrence aided with the temporary immune suppression in the immediate postoperative period associated with rapid weight loss and limited oral intake could be responsible for the formation of the abscess in a partially infarcted spleen from a transient bacteremia.

The signs and symptoms of splenic abscess are nonspecific and include the triad of fever, left upper quadrant tenderness, and leukocytosis $[22,23]$. Its rarity and the nonspecific clinical presentation could make the diagnosis difficult and could be easily confused with leakage and subdiaphragmatic abscess [2].

CT scan is considered as the gold standard for definitive diagnosis of the splenic abscess [2,21,22]. Ultrasound can also demonstrate the characteristics of the splenic abscess [23]. Both of these imaging studies have a sensitivity of $98 \%[18,24]$.

The classical treatment of a splenic abscess is splenectomy with antibiotics therapy. However, more recent reports showed increasing trends towards less invasive procedures, including laparoscopic drainage and splenectomy and spleen preserving protocols such as percutaneous imaging-guided drainage especially in a solitary splenic abscess $[19,25]$

\section{Conclusion:}

Partial splenic demarcation and infarction are not uncommon and have been reported following LSG. It may have no clinical relevance in the vast majority of patients and it usually resolved spontaneously. However, rarely, it may get infected either via direct extension, from a nearby infection, or through hematological spread resulting in a splenic abscess. Splenic abscess complicating LSG is extremely rare with only a few cases reported in the literature. Most of the reported cases of post-LSG splenic abscesses were associated with gastrectomy leakage, although few cases were reported in absence of a documented leak.

The rarity of the condition and the nonspecific symptoms make the preoperative diagnosis difficult and confusable with leakage from the staples line and sub-diaphragmatic abscess. CT scan is considered the gold standard for definitive diagnosis with very high sensitivity.

Solitary abscesses may be treated successfully by laparoscopic or percutaneous drainage with combination with antibiotic therapy. Splenectomy is indicated in the presence of multiple abscesses or when other methods failed.

\section{Conflict of Interest}

None declared

\section{References:}

1. Hess DS, Hess DW (1998) Biliopancreatic diversion with a duodenal switch. Obes Surg; 8: pp. 267-282.

2. Shanti Hiba, Firas Obeidat (2017) "Splenic Abscess Secondary to Sleeve Gastrectomy Leak. Laparosc Endosc Surg Sci; 24(2):50-53.

3. Chung AY, Thompson R, Overby DW, Duke MC, Farrell TM (2018) "Sleeve Gastrectomy: Surgical Tips". Journal of Laparoendoscopic \& Advanced Surgical Techniques. Part A. 28(8): 930-937.

4. Spiegel HU, Skawran S (2011) "From longitudinal gastric resection to sleeve gastrectomy--revival of a previously established surgical procedure". Journal of Gastrointestinal Surgery. 15 (1): 219-228.
5. Gumbs AA, Gagner M, Dakin G, Pomp A (2007) Sleeve gastrectomy for morbid obesity. Obes Surg; 17: pp. 962-969.

6. Akkary E, Duffy A, Bell R (2008) Deciphering the sleeve: technique, indications, efficacy, and safety of sleeve gastrectomy. Obes Surg; 18: pp. 1323-1329

7. Frezza EE, Reddy S, Gee LL, Wachtel MS (2009) Complications after sleeve gastrectomy for morbid obesity. Obes Surg. Jun; 19 (6):684-687.

8. Aurora AR, Khaitan L, Saber AA (2012) Sleeve gastrectomy and the risk of leak: a systematic analysis of 4,888 patients. Surg Endosc ; 26:1509-15015.

9. Stamou KM, Menenakos E, Gomatos IP, Panousopoulos SG, Smparounis S et al (2011) Clinical implications of sleeve gastrectomy as a source of spleen infarction or ischemia. Obes Surg ;21:1490-1493.

10. Dhanasopon AP, Lewis CE, Folek JM, Dutson EP, Mehran A (2009) Splenic infarct as complication of sleeve gastrectomy. Surg Obes Relat Dis;5:626-629.

11. Stamou KM, Menenakos E, Gomatos IP, Panousopoulos SG, Smparounis S et al (2011) Clinical implications of sleeve gastrectomy as a source of spleen infarction or ischemia. Obes Surg; 21:1490-1493.

12. Martínez DG, Sánchez AW, García AP (2008) Splenic abscess after laparoscopic Nissen fundoplication: a consequence of short gastric vessel division. Surg Laparosc Endosc Percutan Tech; 18:82-85.

13. Dhanasopon AP, Lewis CE, Folek JM, Dutson EP, Mehran A (2009) Splenic infarct ascomplication of sleeve gastrectomy. Surg Obes Relat Dis. Sep-Oct; 5(5):626-629.

14. Salvi PF, Stagnitti F, Mongardini M, Schillaci F, Stagnitti A. Splenic infarction, rare cause of acute abdomen, only seldom requires splenectomy. Case report and literature review. Ann Ital C 32.

15. Nores M, Phillips EH, Morgenstern L, Hiatt JR (1998) The clinical spectrum of splenic infarction. Am Surg 1998; 64: 182 188.

16. Antopolsky M, Hiller N, Salameh S, Goldshtein B, Stalnikowicz R (2009) Splenic infarction: 10 years of experience. Am J Emerg Med 27: 262-265.

17. Arslan F, Batirel A, Tabak F, Mert A (2011) Splenic abscess caused by MRSA developing in an infarcted area: case report and literature review. J Infect Chemother 17:851-4.

18. Ooi LL, Leong L (1997) Splenic abscesses from 1987 to 1995. Am J Surg ; 174:87-93.

19. Carbonell AM, Kercher KW, Matthews BD, Joels CS, Sing RF et al (2004) Laparoscopic splenectomy for splenic abscess. Surg Laparosc Endosc Percutan Tech ;14:289-91.

20. Miguel E. Cervera-Hernandez, Dieter Pohl (2017) Splenic abscess caused by Streptococcus anginosus following laparoscopic sleeve gastrectomy: a case report of a rare complication of bariatric surgery, Journal of Surgical Case Reports, Volume 2017, Issue 4, April 2017, rjx072.

21. Avulov V, McQuillen DP, Mizusawa M (2014) Splenic abscess after sleeve gastrectomy. SAGES Metab Obes Arch; 7:71.

22. Singh Y, Cawich S, Aziz I, Naraynsingh V (2015) Delayed splenic abscess after laparoscopic sleeve gastrectomy. BMJ Case Rep. Feb 17;2015.

23. Schiavo L, Scalera G, De Sena G, Ciorra FR, Pagliano P et al (2015) Nonsurgical management of multiple splenic abscesses in an obese patient that underwent laparoscopic sleeve gastrectomy: case report and review of literature. Clin Case Rep. Oct; 3(10):870-4. 
24. Chang KC, Chuah SK, Changchien CS, Tsai TL, Lu SN et al (2006) Clinical characteristics and prognostic factors of splenic abscess: a review of 67 cases in a single medical center of Taiwan. World J. Gastroenterol. 12:460-464.
25. Zerem E, Bergsland J (2006) Ultrasound guided percutaneous treatment for splenic abscesses: The significance in treatment of critically ill patients. World J Gastroenterol ;12:7341-7345.
This work is licensed under Creative Commons Attribution 4.0 License

To Submit Your Article Click Here: Submit Manuscript

DOI: $10.31579 / 2690-1919 / 019$
Ready to submit your research? Choose Auctores and benefit from:

* fast, convenient online submission

* rigorous peer review by experienced research in your field

* rapid publication on acceptance

* authors retain copyrights

* unique DOI for all articles

* immediate, unrestricted online access

At Auctores, research is always in progress.

Learn more www.auctoresonline.org/journals/clinical-medical-reviewsand-reports 